\title{
L'EXAMEN MICROSCOPIQUE DES FROMAGES (1)
}

\author{
Par le Professeur Otakar LAXA
}

Dans l'analyse des fromages, l'observateur n'emploie le mieroscope que dans peu de cas. La détermination des miettes, l'addition des pommes de terre, l'observation de la qualité des moisissures, c'est presque uniquement pour cela que le microscope est employé.

Le but de ce travail est de présenter un essai microscopique de la pâte des fromages, en d'autres termes, d'étudier la structure microscopique de ceux-ci.

Dans la fromagerie, on travaille avec des protéines, c'est-à-dire des colloïdes, caséine et paracaséine, que nous coagulons soit par l'acidification, soit par la présure.

La manière dont on effectue la coagulation de ces colloïdes, les soins apportés au coagulum sont les causes premières des différences obtenues entre les produits finals, principalement lorsque la préparation utilise la présure. On peut supposer que les petites parties de la caséine (lactoconies) dans le lait ont une forme favorable à leur agglutination. La présure et les acides aussi changent la situation de la caséine dans le même sens, car il se produit comme une contraction. qui entraine la caséine de ces parties fines de leur soudure. Le moulage du caillé et la maturation des fromages frais amènent de nouveaux changements de la pâte. Aussi peut-on penser à priori que l'examen microscopique permettra de connaître beaucoup de changements de la easéine ou de la paracaséine au cours de la préparation des fromages. On en pourra tirer quelque chose relativement à l'influence du mode de coagulation, du moulage et de la maturation.

La technique employée pour l'observation microscopique est la suivante : on coupe avec un scalpel une tranche très mince du fromage ou de la caillebotte compacte ; quand il s'agit de fromage mou ou de caillebotte molle, on utilise un petit morceau de la grosseur d'une tête d'épingle. On pose l'objet sur la lame, on le couvre d'une lamelle, on renverse le tout et on presse fortement. On ne doit pas employer d'eau, parce qu'elle peut dissoudre certains produits de la décomposition, qui échapperaient ainsi à l'observation. S'il le faut, on met la substance dans la glycérine. L'observation est exécutée avec le grossissement $300 / 1$, exceptionnellement $100 / 1$, parfois $800 / 1$.

La caillebotte et le caillé obtenus du lait écrémé ont une structure très simple. On voit une masse homogène de grains très fins, çà et là percée de, cavités ou de petites crevasses où loge du patit-lait (fig. 1 et 2) et qui forment dans la caillebotte légèrement pressée une sorte de mosaïque.

(1) Ce travail a été présenté le 5 septembre 1925, à l'Académie d'Agriculture de Tchécoslovaquie, à Prague. 
La caillebotte et le caillé frais,préparés avec du lait non écrémé,a une structure toute différente. Les globules gras empêchent que la

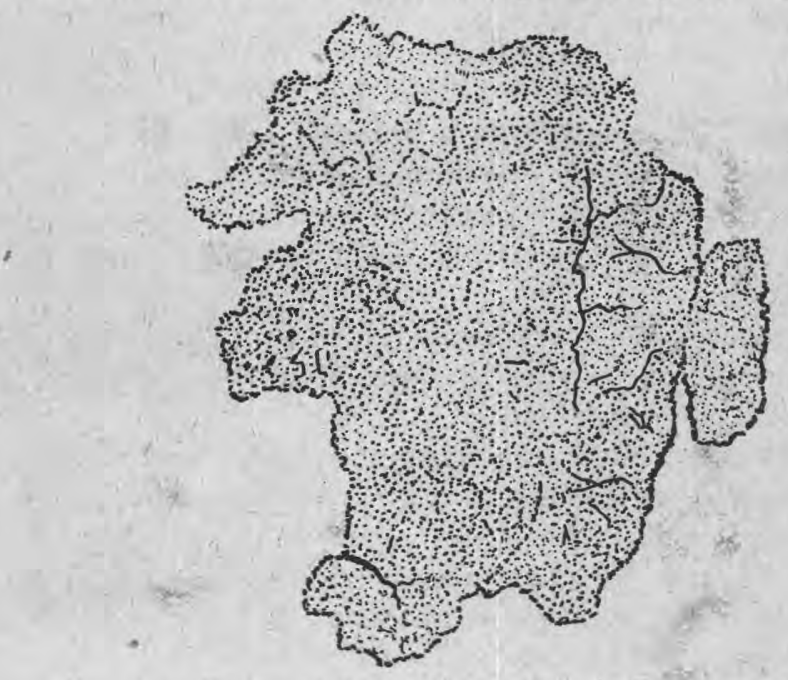

F16. I. - Fragment de caillebolte maigre. Grossissement I : 3oo.

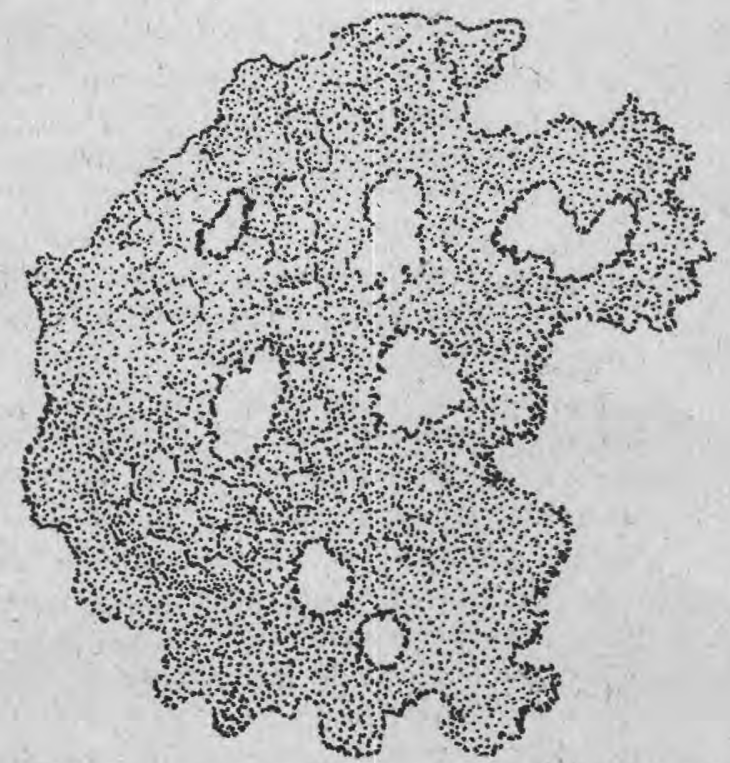

F1G. 2. - Fragment de caillé du lait écrémé, Grossissement 1 : 3oo.

caséine ou la paracaséine se contractent et forment une masse compacte et homogène. Le coagulum des albuminoïdes se contracte autour des globules gras et il en résulte une structure à gros grains. 
La masse est percée de cavités où loge le petit-lait (fig. 3). Nous trouvons une pareille structure à gros grains dans les fromages mous à la crème, par exemple ehez les petits suisses, gervais et brynze (fig. 6 et 7).

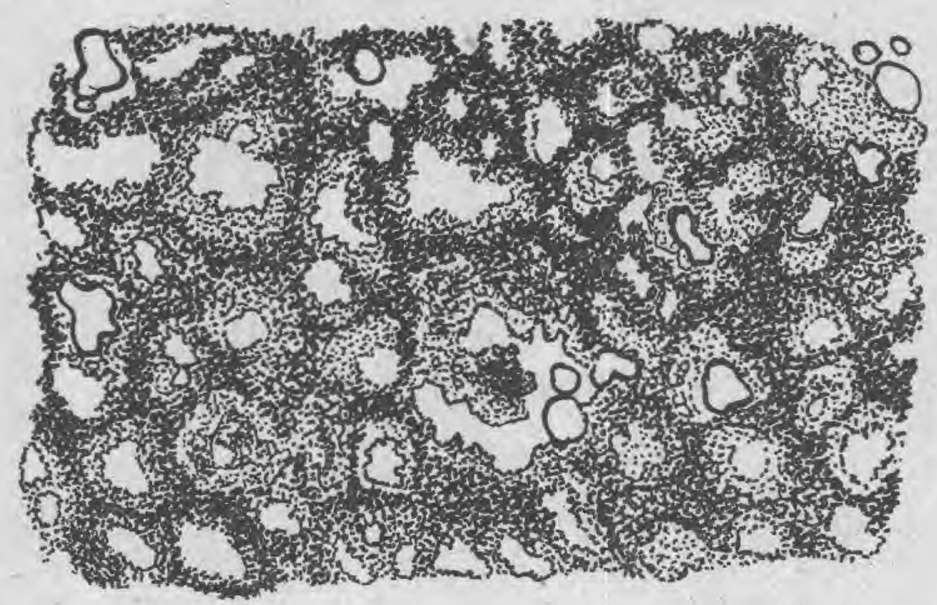

Fts. 3. - Fragment de caillé du lait gras, Coloré. Grossissement i : 3oo.

La grandeur des cavités remplies de petit-lait dépend du temps du caillage, e'est-à-dire de la quantité de présure employée et du degré de la température. Sur les figures 4 et 5 on voit nettement quelle influence a le temps du caillage sur la grandeur et le nombre des cavités de petit-lait.

Le lait pour cette expérience était fortement coloré au violet de gentiane et divisé en deux parties. Le temps d'emprésurage de l'une était de une heure, celui de l'autre de un quart d'heure. Le caillé était coupé puis laissé à égoutter spontanément. On a pris un échantillon du fromage et on l'a observé au microscope avec un grossissement léger. Alors que le fromage du caillage d'une heure présentait de nombreuses et des vastes cavités remplies de petit-lait, le fromage avec caillage d'un quart d'heure d'emprésurage avait des cavités en moins grand nombre et plus petites. On voit aussi l'effet de l'emprésurage prolongé chez le fromage impérial, sorte de fromage gervais (fig. 6). La masse est percée de nombreuses et relativement vastes cavités qui sont très souvent remplies par des gouttes de graisse.

Toutes les opérations qui augmentent la contraction de coagulum, e'est-à-dire le chauffage et la pression, engendrent avec de la matière première non écrémée la structure à gros grains. L'influence du chauflage est, avec l'observation microscopique, plus évidente que celle de la pression, eomme le démontre l'essai suivant. 


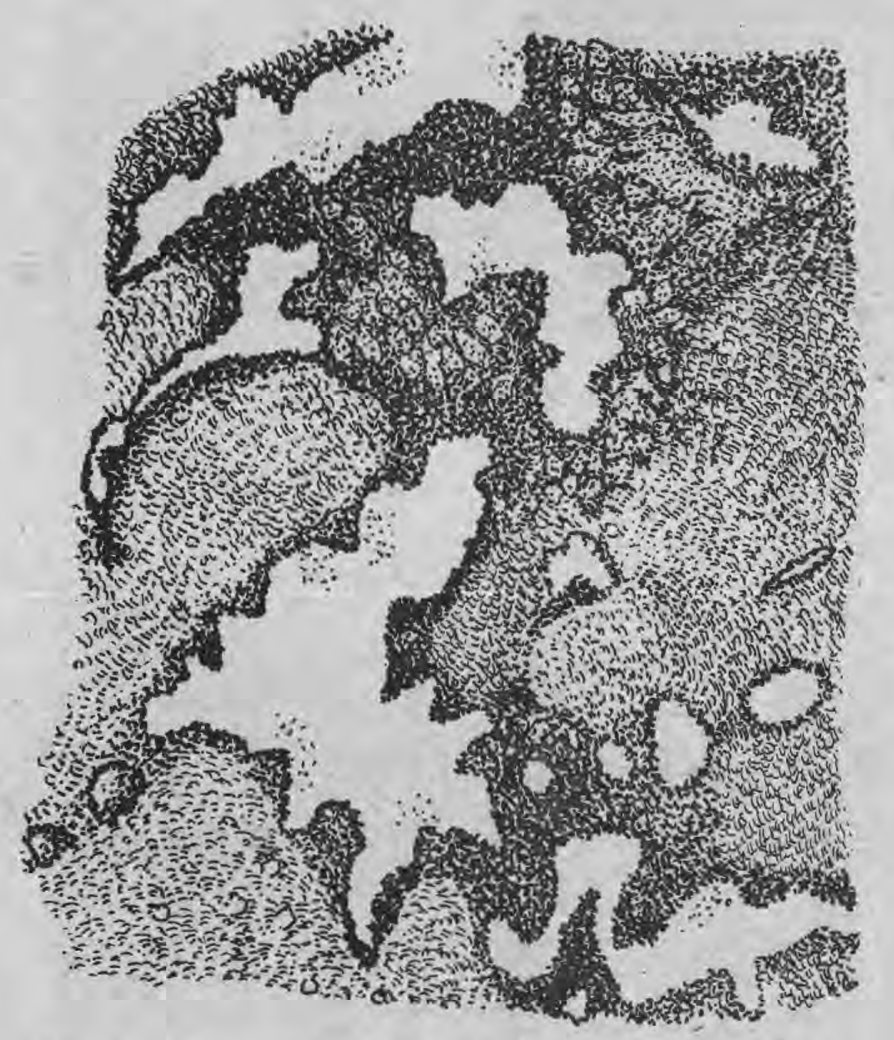

FIs. 4. - Fragment de caillé gras du lait coloré par le violet de gentiane. Coagulation $t$ heure. Gross. I : $t 00$.

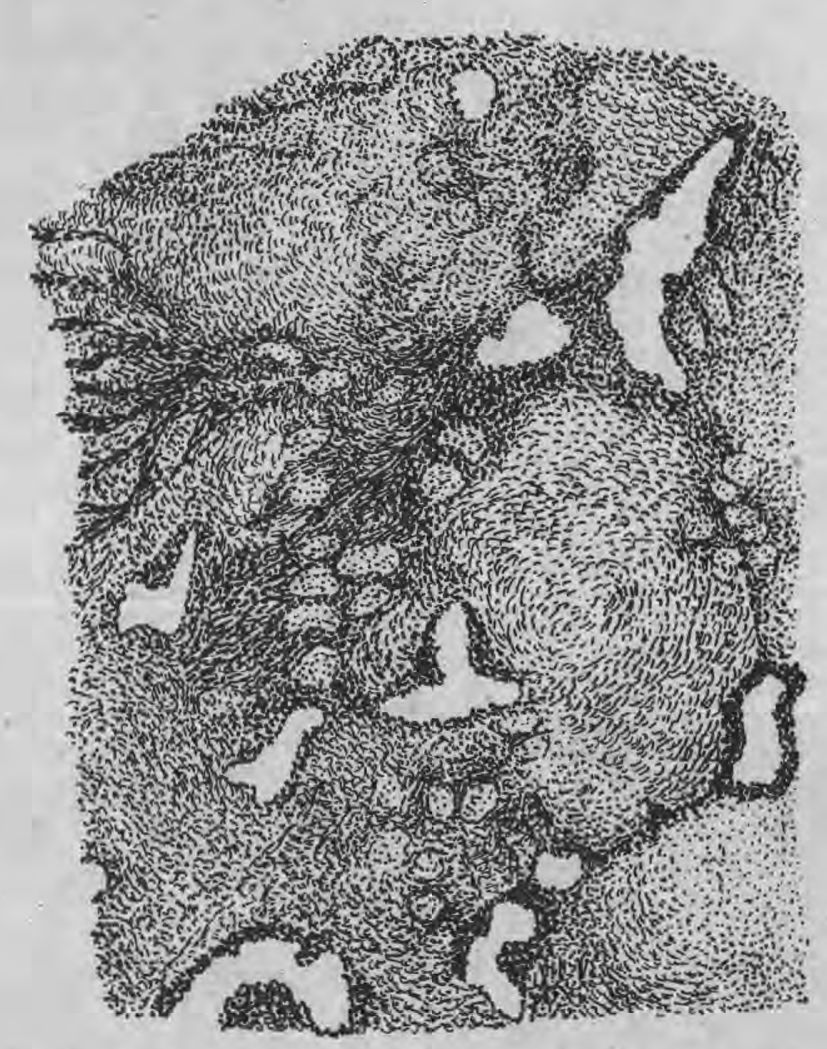

Fro. 5. - Fragment de caillé gras du lait coloré par le violet de gentiane, Coagulation $1 / 4$ d'heure. Gross, 1 : 100. 


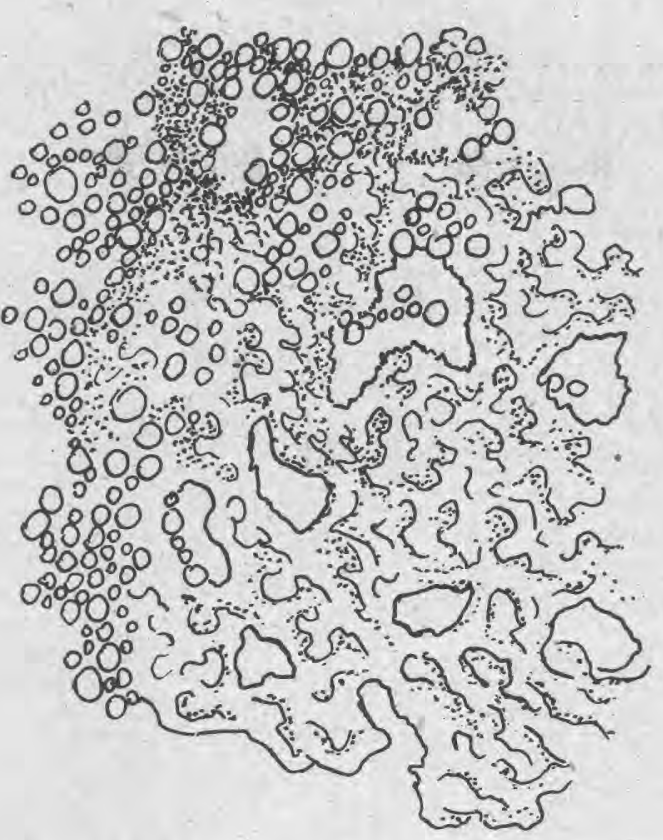

FIG. 6, - Fragment de fromage « impérial » (gervais). fromage surchoix, non coloré. Grossissement r : 3 oo.

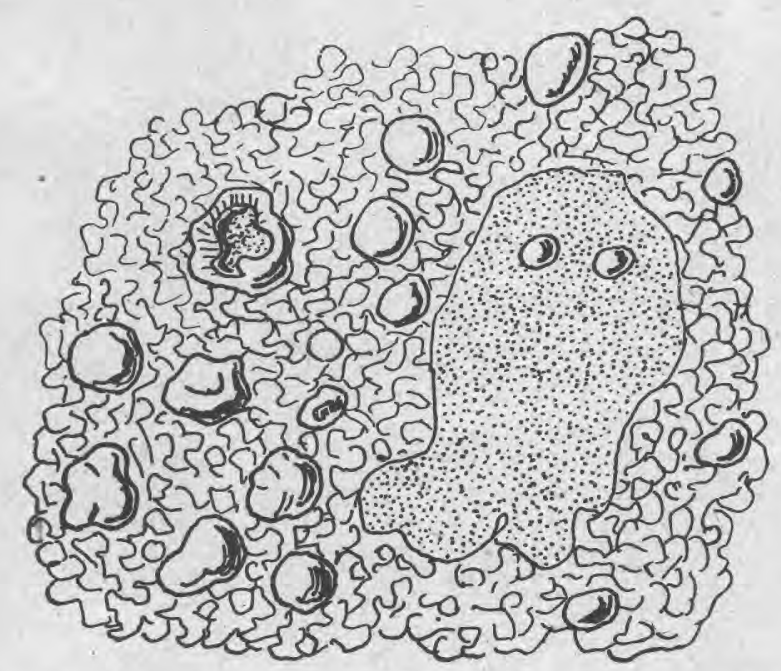

Fig. 7. - Fragment de brynza (fromage surchoix de lait ovin) falsifié par la caillebotte maigre. Les grains de Ia leucine. Non coloré. Grossissement 1 : 300 . 


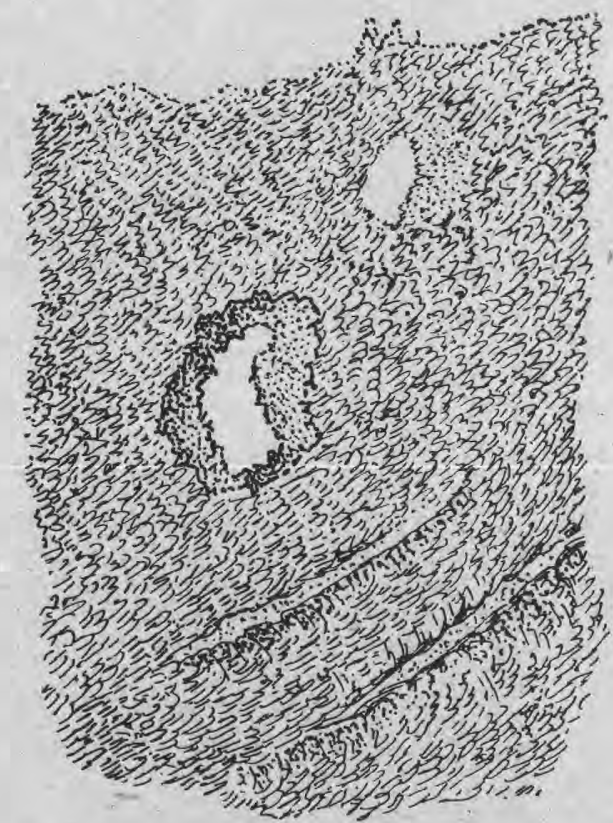

FIG, 8. - Fragment de caillé maigre chauffé a $47^{\circ} \mathrm{C}$, non pressé et non coloré. Grossissement $x$ : $30 o$.

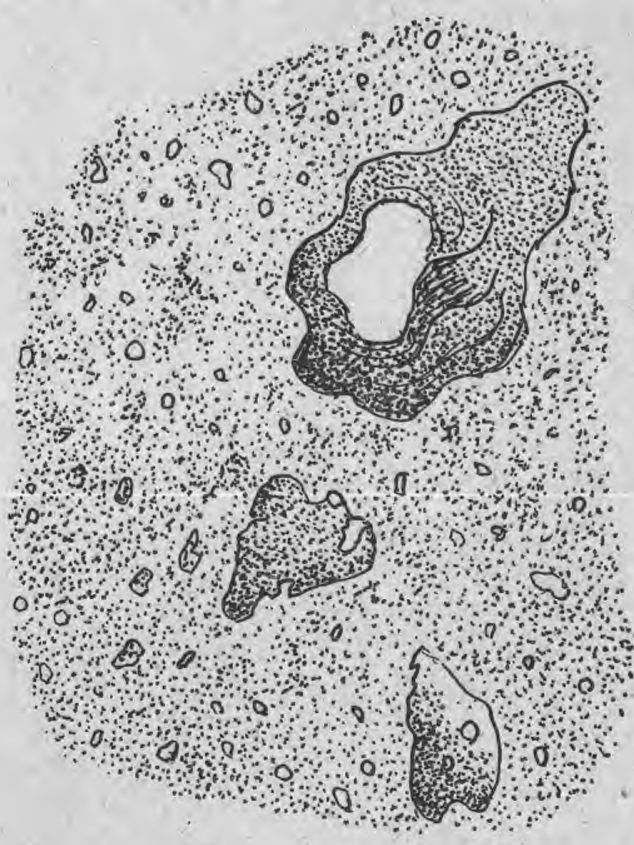

Fig. 9.- Fragment de caillé maigre chauffé à $47^{\circ} \mathrm{C}$, pressé et non coloré. Grossissement I : 300. 
Le lait écrémé, dont l'emprésure a demandé une demi-heure, a donné un caillé qu'on a coupé et rompu, puis chauffé à une température $47^{\circ} \mathrm{C}$. La préparation faite du fromage ainsi obtenu montre distinctement (voir fig. 8) que la masse il est vrai est à grains fins, mais qu'elle est aussi plus compacte que celle du même fromage dont la pâte n'a pas été chauffée (fig. 2). La pression n'a pas apporté les changements plus évidşıts (fig. 9), tout au plus, la masse possède-t-elle de plus nombreuses crevasses.

Le lait non éerémé, emprésuré à une température $35^{\circ} \mathrm{C}$ en une demi-heure, a donné un caillé qui de même que le précédent a été coupé, rompu et réchauffé à $47^{\circ} \mathrm{C}$. Du fromage frais ainsi obtenu, on a fait une préparation sur laquelle (fig. 10) on peut reconnaître que la masse est à gros grains.

Le fromage garmond (fig. 11) a aussi une structure semblable. On peut déduire de cela que, pendant sa préparation, on a utilisé le chauffage.

Le pressage du caillé réchauffé n'a fait que diminuer les cavités du petit-lait ; par ailleurs il n'a effectué aucun changemènt (fig. 12). On rencontre une pareille structure à gros grains chez le fromage d'Emmenthal (fig. 13), d'Edam (fig. 14) et chez le fromage trappiste (fig. 15), garmond (fig. 16), même chez le chester (fig. 17). Nous trouvons spéeialement dans l'ostepek (fig. 19) et dans le parmesan (fig. 18) une structure prononcée à gros grains rappelant presque les cellules de la cire pressée des abeilles comme suite du chauffage à température élevée.

Le rompage du caillé a pour résultat la division de la masse en petits morceaux, qui deviennent visibles au microscope quand le fromage est préparé après chauffage du caillé (fig. 16, 20).

Le microscope nous montre aussi si le fromage a subi la maturation et à quel degré. Aussitôt que sous l'action des ferments commencent dans le fromage les changements protéolytiques qui amènent la décomposition en albuminoses, peptones et acides aminés de la caséine et de la paracaséine, il se produit certains changements dans la structure du fromage. Ces changements sont causés par les produits de la décomposition, principalement par les acides aminés qui ont pris naissance au cours de la maturation des fromages. Dans la pâte de fromage se montrent des grains, le plus souvent d'une structure radiaire. On'a pris longtemps ces grains pour de la tyrosine. Cette substance est un produit de la caséine au cours de la décomposition protéolytique de celle-ei et de son insolubilité dans l'eau témoigne en faveur la possibilité de sa cristallisation dans la pâte du fromage ; une partie de tyrosine a besoin en effet pour se dissoudre de 2450 parties d'eau $20^{\circ} \mathrm{C}$.

Les grains qui se trouvent régulièrement dans les fromages qui 


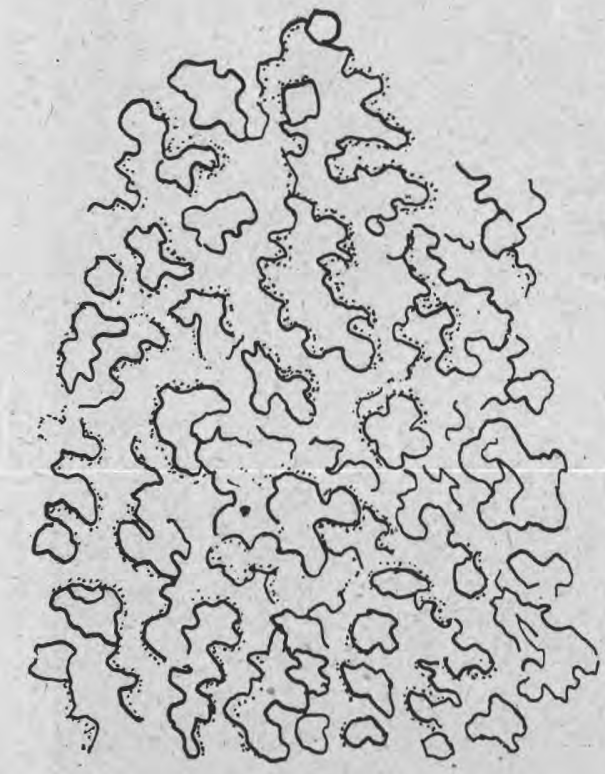

FIG. ro. - Fragment de caillé gras, chauffé à $47^{\circ} \mathrm{C}$.

Non coloré. Grossissement 1 : 300.

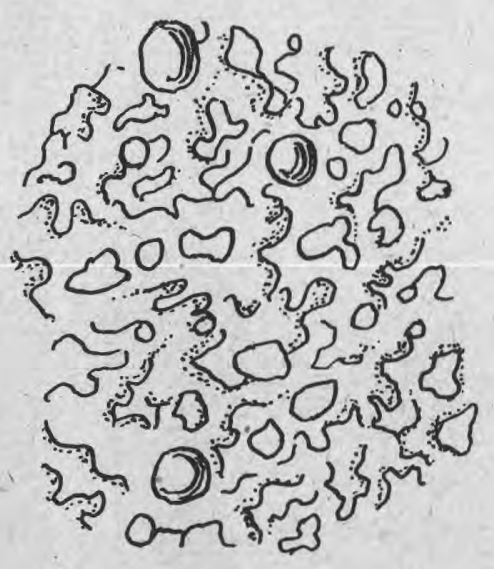

Fig. II, - Fragment de fromage salami. Les grains de la leucine.

Non coloré. Grossissement i : 300 .

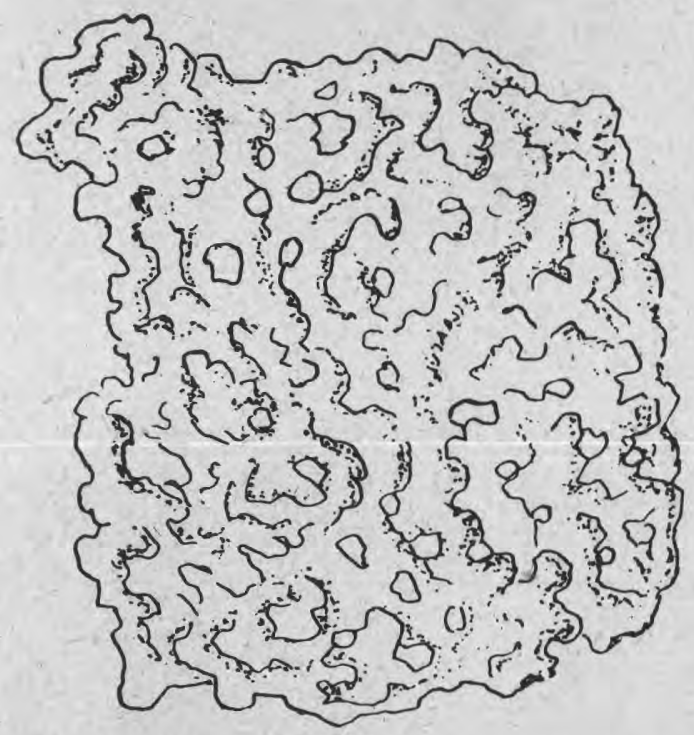

FIG. 12. - Fragment de caillé gras, chauffé à $47^{\circ} \mathrm{C}$ et pressé.

Non coloré. Grossissement 1 : 3oo, 


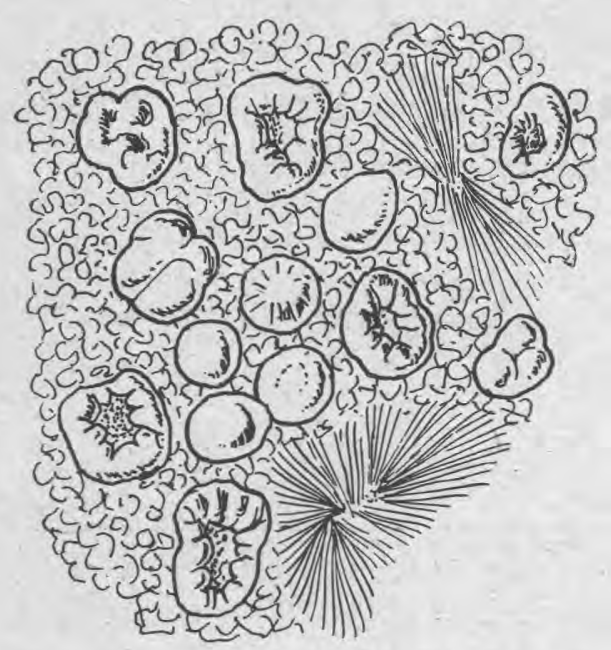

Fig. ı3. - Fragment de fromage de gruyère, Les grains de la leucine et les druses de tyrosine. Grossissement 1 : 300.

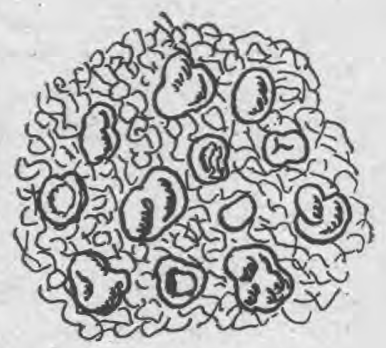

Fig. 14, - Fragment de fromage d'Edam. Gross, x : 300.

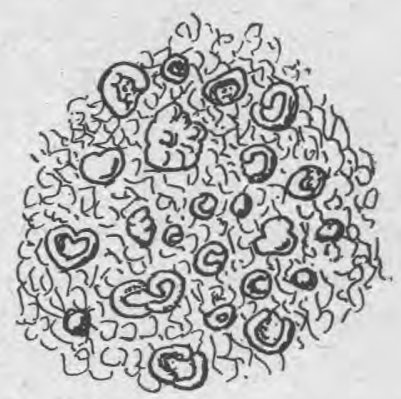

Fig. 15. - Fragment de fromage trappiste. Non coloré. Grossissement I : 300 .

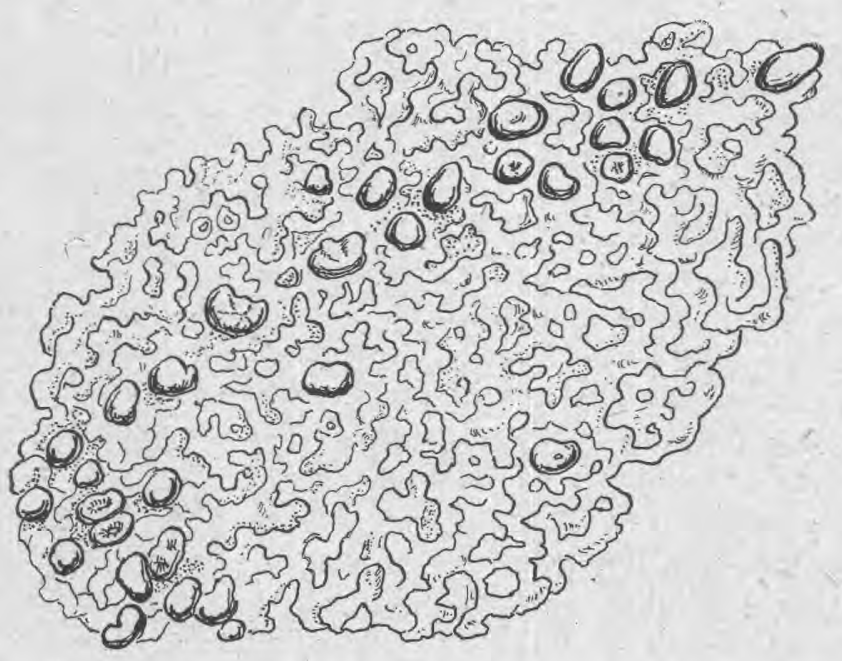

Fis. r6. - Fraónent de fro nage b.llelay (tête de moine). Non coloré. Grossissement 1 : 3oo. 
mûrissent et dans les fromages affinés, je les ai pris aussi tout d'abord pour la tyrosine, mais plus tard, je me suis persuadé que ce n'était pas juste. Il me semblait curieux que, même les fromages peu mûrs, par exemple le garmond, ont de nombreux grains, quoique la décomposition de la paracaséine dans ces fromages fut peu avancée. Et pourtant la tyrosine ne prend naissance que lorsque la décomposition est plus avancée et elle se forme en moindre quantité. Au contraire, la leucine se forme pendant la décomposition très facilement et en quantités plus grandes. Mais la leucine est relativement aisément soluble dans l'eau. Une partie de la leucine se dissout dans 45 parties d'eau à $10^{\circ} \mathrm{C}$. Il était donc nécessaire de déceler le caractère des grains, qui se trouvent régulièrement dans les fromages.

Ces grains sont caractéristiques, en disque ou à trois lobes, le plus souvent ayant la forme de coquilles, de petits lobes d'oreille; leur grandeur varie de $12 \mu-40 \mu$ (fig. 13-20, 25-30).

La tyrosine chimiquement pure donne une agglomération d'aiguilles, qui sont liées en forme de balai (fig. 21). La tyrosine donne la réaction du biuret.

La leucine forme des agglomérations d'aiguilles très fines, qui sont unies dans des sphérocristaux en forme de lentilles, très souvent avee une cavité au milieu (fig. 22). La leucine ne donne pas la réaction du biuret.

On ne peut pas exécuter par voie mierochimique la réaction du biuret, parce que la coloration violette ou bleue, si la réaction est négative, disparaît par transparence à la lumière, il ne reste qu'à recourir à la forme d'agglomération de cristaux. La leucine pure, préparée par voie chimique en partant de rabotures de corne, donne les mêmes sphérocristaux qu'on observe dans les grains des fromages; au contraire, les cristaux de la tyrosine pure donnent des formes de balai. C'est sur quoi je base ma conviction que les grains dans les fromages sont constitués de leucine. La présence de nombreux grains de leucine dans les fromages n'est pas en opposition avec sa solubilité relativement facile. Si nous considérons que, par exemple, le fromage d'Emmenthal, faiblement mûr, contient environ $34 \%$ d'eau et $1,5 \%$ d'acides aminés, principalement de la leucine, nous devons reconnaître que la majeure partie de cette substance sera précipitée, parce que la quantité de la leucine exprimée ci-dessus exige pour se dissoudre complètement $67,50 \%$ d'eau. Egalement, dans le garmond avec $43 \%$ d'eau et $2 \%$ d'acides aminés, doit-il y avoir des graines de leucine, puisque pour la dissolution complète il faudrait $90 \%$ d'eau.

Le lactate de chaux forme des amas d'aiguilles, qui ressemblent un peu aux grains de leucine (fig. 23). Ces amas affectent plutôt une forme de hérisson, très souvent radiés. J'ai trouvé les mêmes formes 


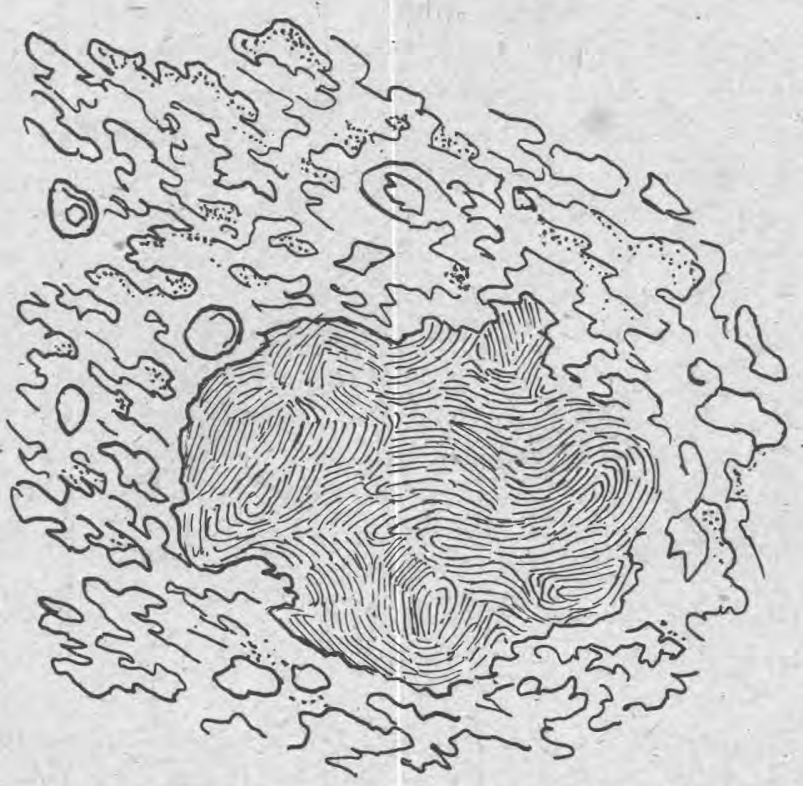

Frg. 17. - Fragment de fromage chester avec une colonie de bactéries. Non coloré. Grossissement r : 300.

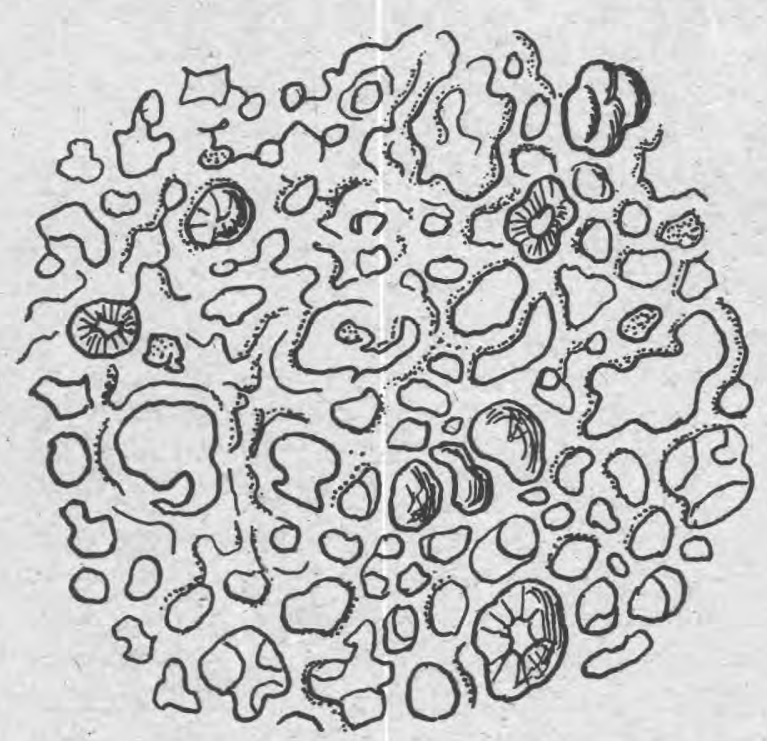

Fra. 18. - Fragment de fromage parmesan (grana). Non coloré. Grossissement $\mathrm{I}: \mathbf{3 0 0}$. 


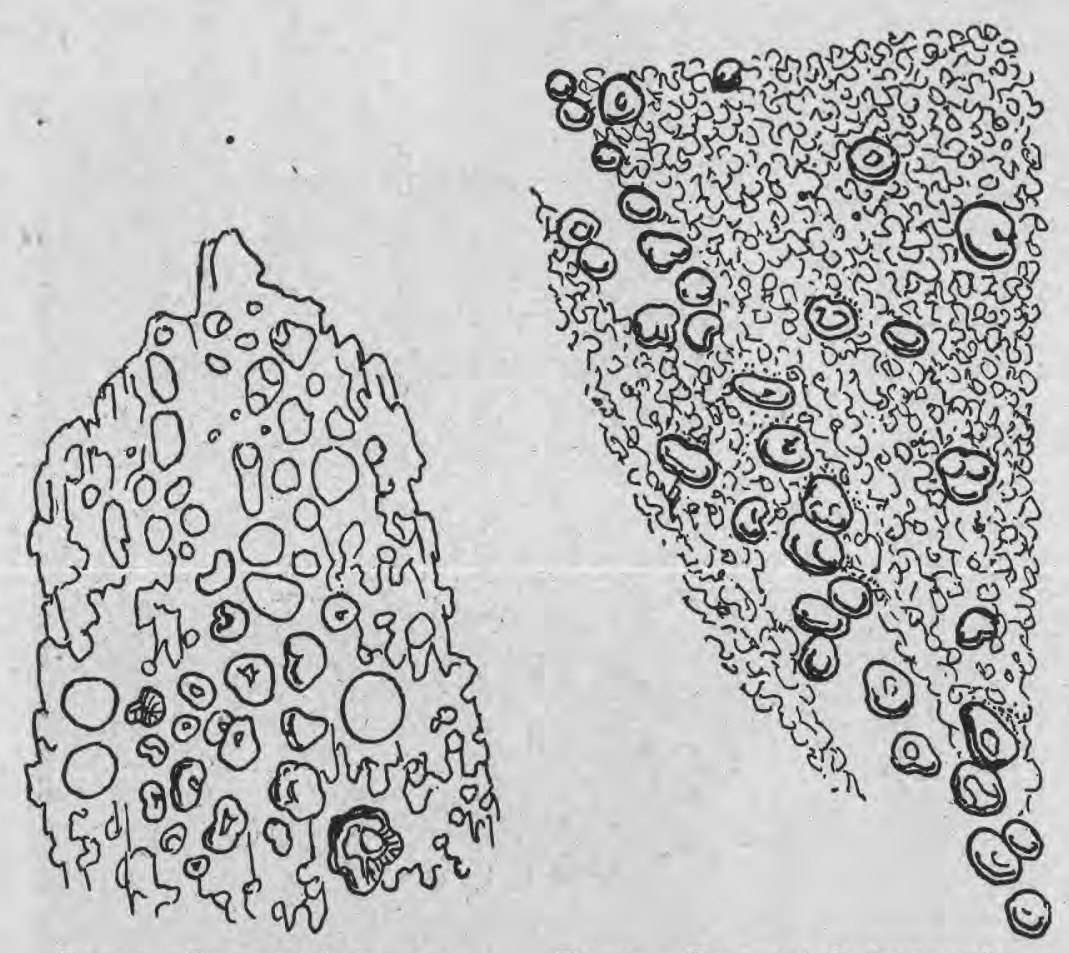

Fig. I9 - Fragment de fromage ostepek. Non coloré. Grossissement I : 3oo.
Fig. 20. - Fragment de fromage de Tilsit. Non coloré. Grossissement 1:3oo.

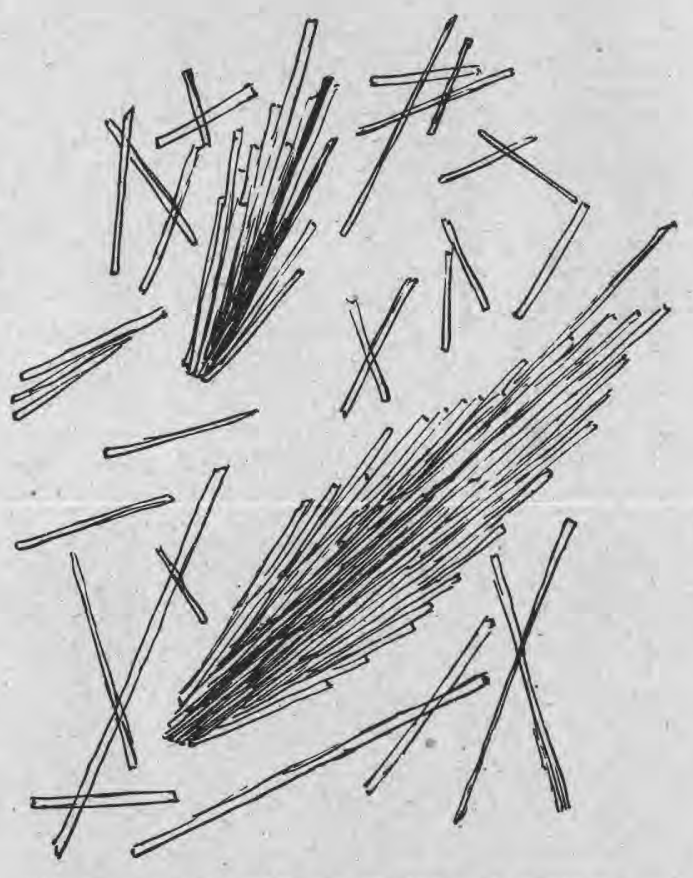

Fig. 21. - Les druses de cristaux de tyrosine, préparés par l'hydratation de raboture de corne. Grossissement $x$ : 300. 
dans des cultures vieilles et sèches de ferments lactiques, accompagnées de peptonisation (fig. 24). Certaines formes trouvées dans le garmond rappellent aussi les druses de lactate de chaux (fig. 25).

Selon le degré de la maturation et la façon dont elle s'est faite, la masse du fromage est pénétrée de grains de leucine, petits ou gros, qui suivent par leur localisation le cours de la maturation. Dans les fromages qui mùrissent d'une manière homogène dans toute la masse, comme dans l'Emmenthal, et les fromages des Pays-Bas, nous voyons les grains répandus dans la pâte d'une manière homogène (fig. 13, 14, 15). Dans ces cas, les grains se forment dans les points où se trouve le plus grand développement des micro-organismes, où le petit-lait se trouve en majeure partie, c'est-à-dire dans le voisinage des cavités. Chez les fromages à morceaux de caillé compact, on peu' trouver des rangs de grains de leucine entre des morceaux de pâte (fig. 16, 20). Les fromages dont la maturation part de la surface, possèdent de nombreux grainș dans la partie molle sous la croûte.

Les fromages avec moisissure dans la pâte, contiennent de gros grains de leucine, et de très nombreux, dans les environs de la végétation de la moisissure. En ces points on peut remarquer des nids de grains de leucine, par exemple dans le roquefort (fig. 26).

Outre la leucine, les autres acides aminés peuvent cristalliser, mais cela n'est pas un phénomène régulier. On peut identifier des formes en balai comme celles des agglomérations de tyrosine (fig. 13), tandis que le caractère chimique des petites plaques et des rhomboèdres (fig. 27 et 28), qui accompagnent la couche mûre dans les fromageons d'Olomouc, ne m'est pas encore connu. Dans la pâte du garmond, généralement dans les lieux de la végétation des moisissures, on y trouve les grains à structure finement radiaire, au caractère gras. Ce sont des acides gras provenant de la graisse du lait sous l'influence des moisissures (fig. 25).

On ne doit pas confondre les agrégats de la graisse du lait, qui se trouvent isolément dans les fromages pasteurisés (fig. 29) avec les précipités d'acides aminés ou avec les colonies de microorganismes qui se logent dans les cavités de la pâte des fromages (fig. 17).

Il a déjà été observé par de nombreux auteurs que les mieroorganismes dans les fromages ne se trouvent pas diffusément répandus dans la pâte, mais qu'ils forment des colonies dans des cavités (Broili-Peterson, Barthel, Weigmann, Ernst). Dans le fromage d'Emmenthal, le chester, le cheddar, on peut trouver tels endroits remplis de colonies de bactéries (fig. 17). Mais même dans les fromages dont la maturation commence par la surface, les microorganismes pénètrent par les crevasses dans les couches plus profondes et y activent la décomposition. Aussi les crevasses de la surface des fromageons d'Olomouc sont pleines de cellules de levures, d'oïdiums 
(3)

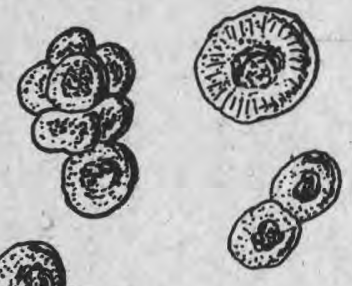

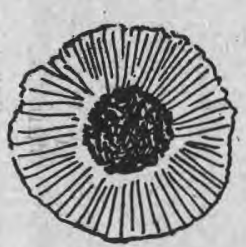

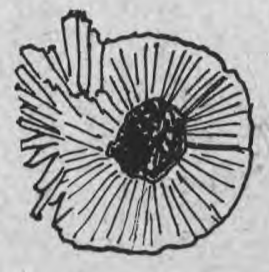

Fig. 22. - Les grains de cristaux de la leucine, préparées par l'hydratation de raboture de corne. Grossissement au-dessus $x$ : roo, au-dessous $1: 300$,

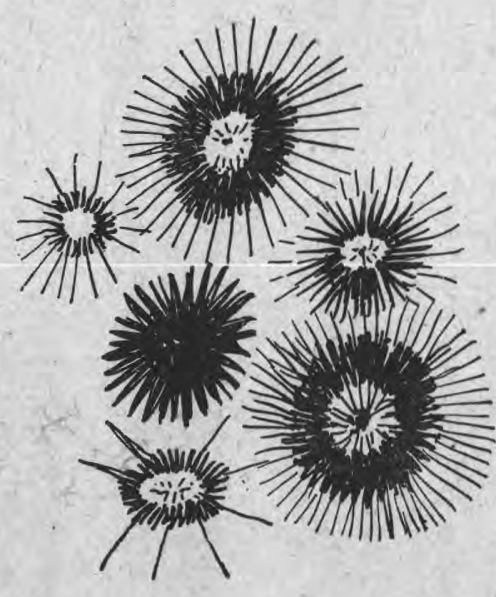

Fig. 23. - Les druses de cristaux de lactate de chaux.

Grossissement 1 : 100.

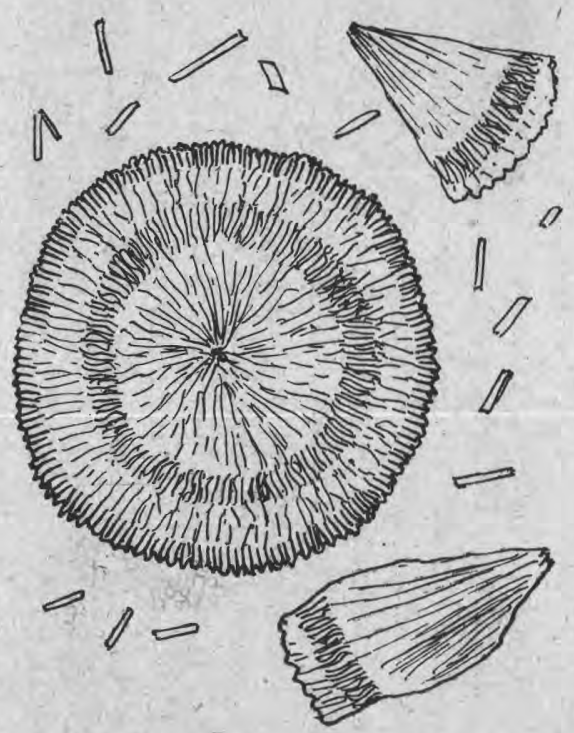

FIG, 24. - Les druses de cristaux de lactate de chaux de culture de lait. Grossissement r : 100. 


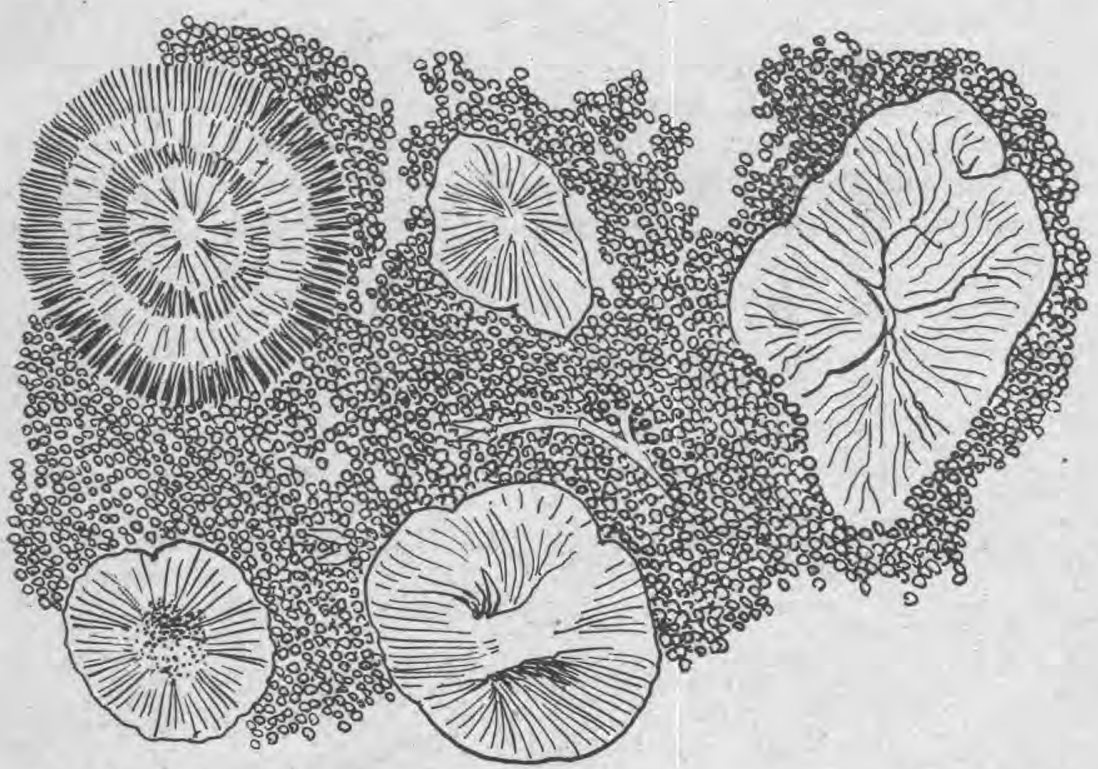

FIG. 25. - Fragment d'une partie verte du fromage gorgonzola avec la formation des agrégats des acides gras et du lactate de chaux (au-dessus à gauche). Non coloré. Grossissement i : 300 .

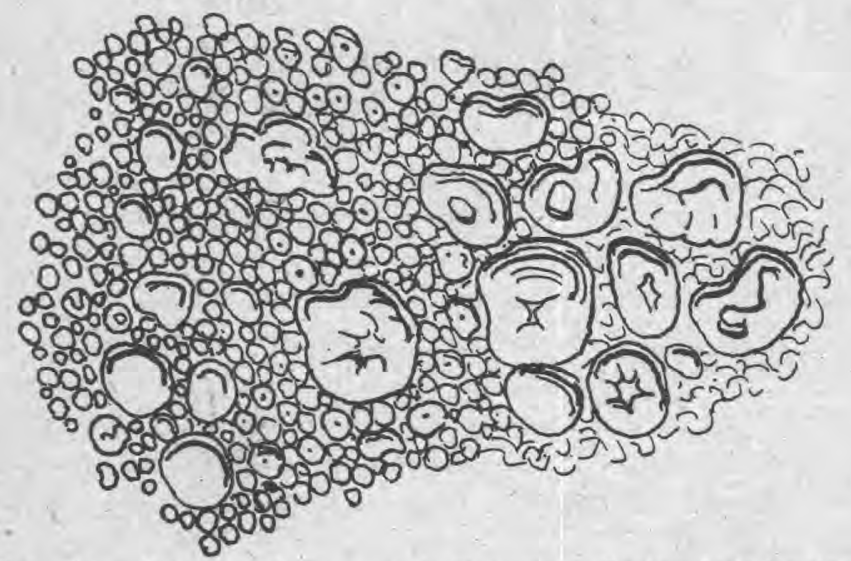

FIG. 26. - Fragment de fromage de Roquefort. Grains de la leucine. Grossissement 1 : 3oo.

et de bactéries. Ces dernières pénètrent même dans les points où l'humidité aide leur végétation et où les levures ne se développent pas par suite du manque d'air (fig. 27 et 28).

Dans les fromages mous et demi-durs qui mûrissent, en outre de la formation des grains de leucine, il effectue aussi un autre changement de toute la masse. 
$\mathrm{Au}$ commencement, la structure des gros grains change de telle façon, que la liaison des coagulats s'atténue et que la masse se réduit en particules isolées, de forme ronde, ovale, qui se colore très facilement avec les couleurs artificielles. Les albuminoses, les peptones, les acides aminés, en général, les matières solubles dans l'eau forment une masse pâteuse, qui en devenant d'un plus petit volume, se

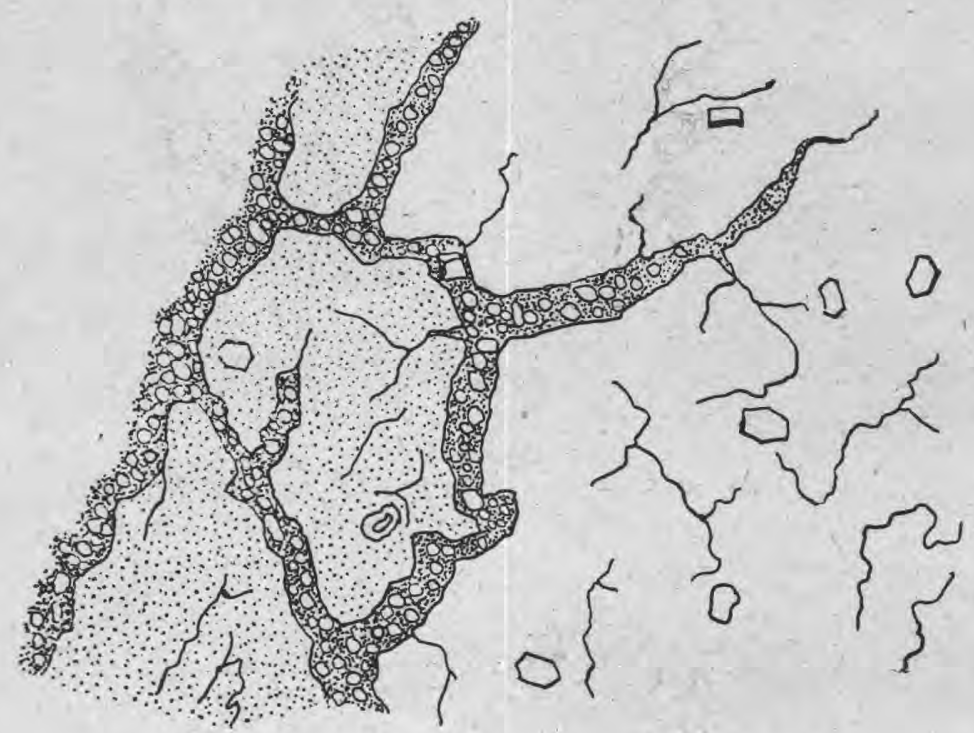

F19. 27. - Fragment de fromageon d'Olomouc (caillebotte maigre) d'une couche superficielle. Non coloré. Grossissement is: 300 .

contractent en formations rondes, ovales, comme on le voit d'après les dessins du fromage affiné (brie, romadour et roquefort) (fig. 30 , $31,26)$.

La couche mûre des fromageons d'Olomouc montre une masse presque sans strueture. La structure à grains fins du caillé et de la caillebotte maigre disparaît, la masse s'unit et elle devient transparente (fig. 27 et 28 ).

Il reste à indiquer dans quelle mesure un praticien peut profiter des observations ci-dessus.

Tout d'abord on peut, dans de nombreux cas, déterminer s'il s'agit de fromage, de caillebotte grasse ou de caillebotte maigre. La structure de la pâte grasse est à gros grains, la pâte maigre est homogène et à grains fins. Il est vrai que cela ne saurait être très général, car les fromages fabriqués avec du lait homogénéisé possèdent leur graisse répandue si finement, que leur structure est aussi à grains 


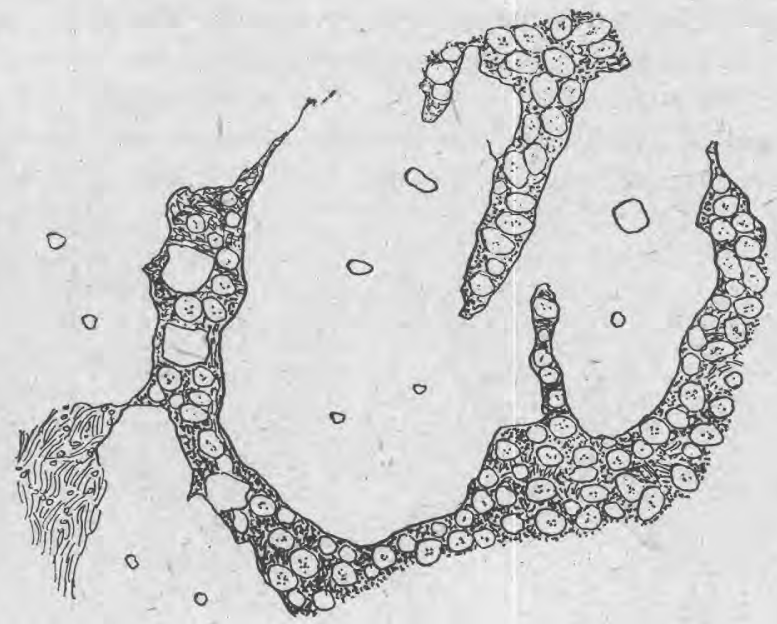

FiG, 28. - Fragment de fromageon d'Olomouc de la couche superficielle. Non coloré. Grossissement I : 800 .

(Les taches claires dans les crevasses signifient généralement les cellules de levures.)

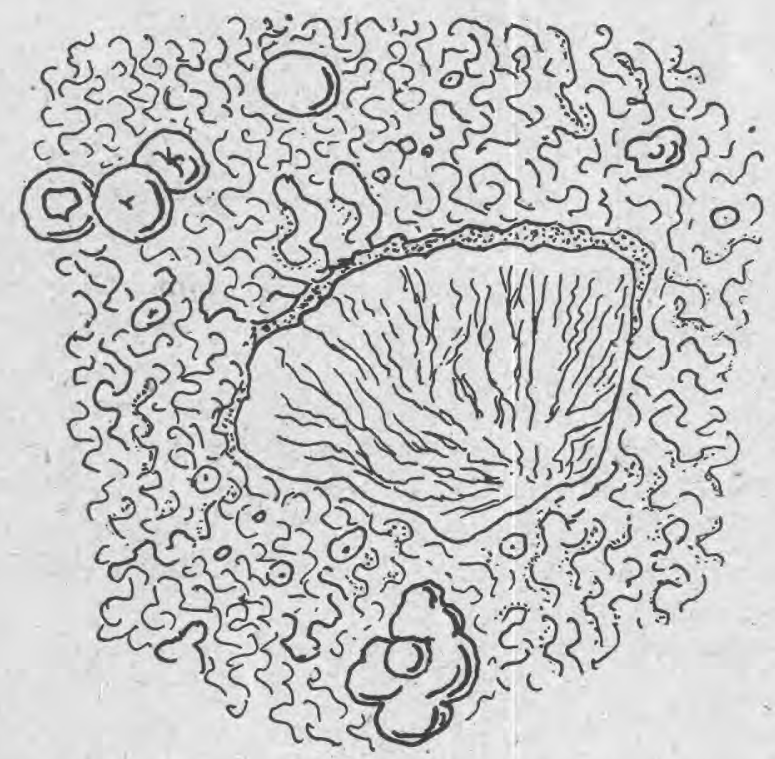

Fig. 20. - Fragment de crème de gruyère (fromage pasteurisé) avec l'agrégat de graisse de lait. Les grains de la leucine. Non coloré.

Grossissement : : 300 . 
fins. Cette remarque n'a de valeur que chez quelques fromages pasteurisés, faits du lait préalablement homogénéisé.

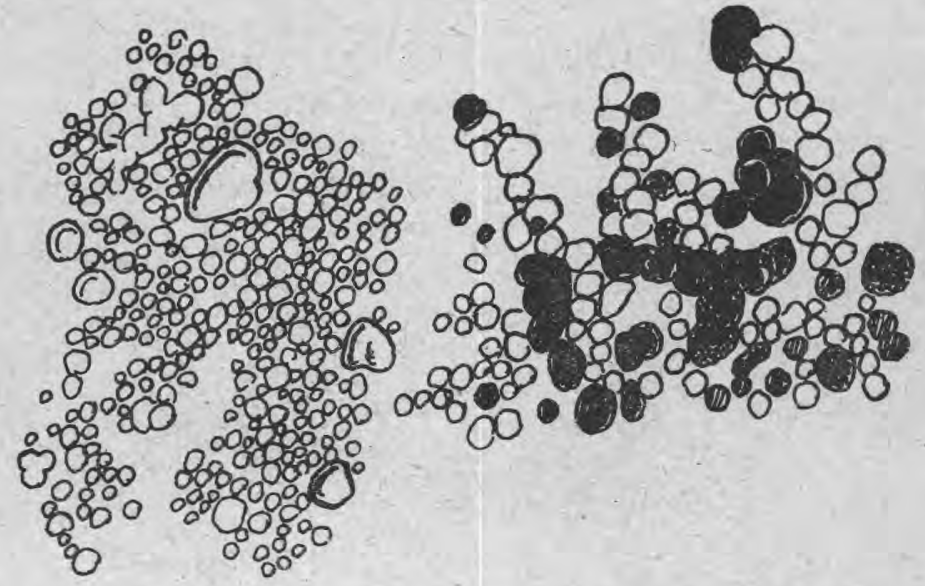

Fig. 3o. - Fragment de la couche maturée du fromage de Brie. A gauche, grossissement $\mathrm{r}: 3 n o$. Non coloré.

A droite, grossissement $ı$ : 800 . Coloré par la fuchsine.

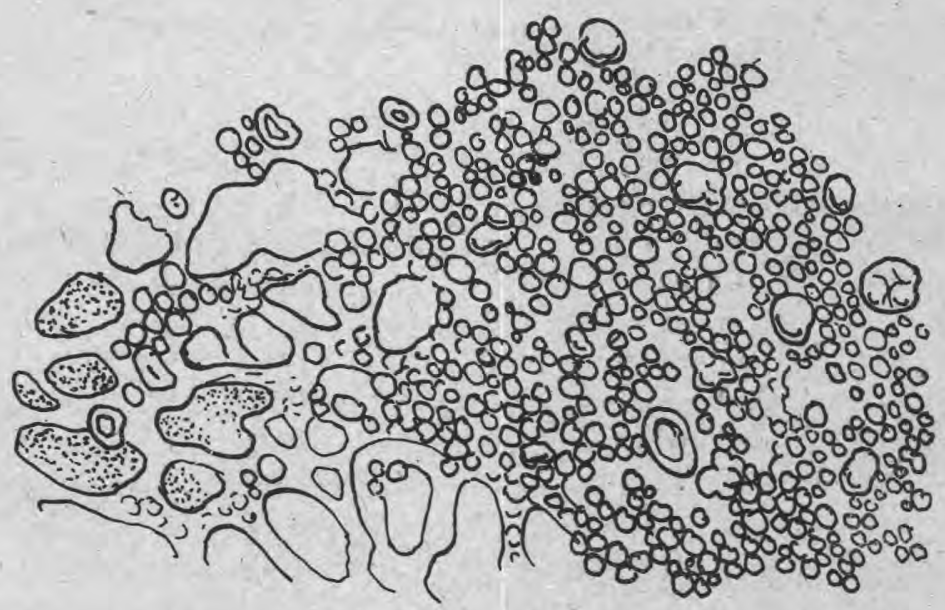

HIG. 31. - Fragment de la couche maturée du fromage romadour. Non coloré. Grossissement I : 300 .

Done dans quelques cas, on peut déceler au microscope s'il s'agit de fromage qui, après la maturation, ont été chauffés à $60-70^{\circ} \mathrm{C}$. Très souvent, on réussit à trouver dans la masse de tels fromages 
des cavités remplies d'agrégats de graisse solide, laquelle s'était séparée pendant le ehauffage de la pâte. Les fromages? de lait homogé néisé possèdent aussi une masse percée de cavités' vides avec des contours ronds et précis, par suite du chauffage de la] pâte du fromage (fig. 32).

Par le microscope, on peut aussi reconnaître le degré de la maturaLion du fromage d'après les grains de la leucine.

Enfin, par le microscope, on peut déceler l'addition de eaillebotte

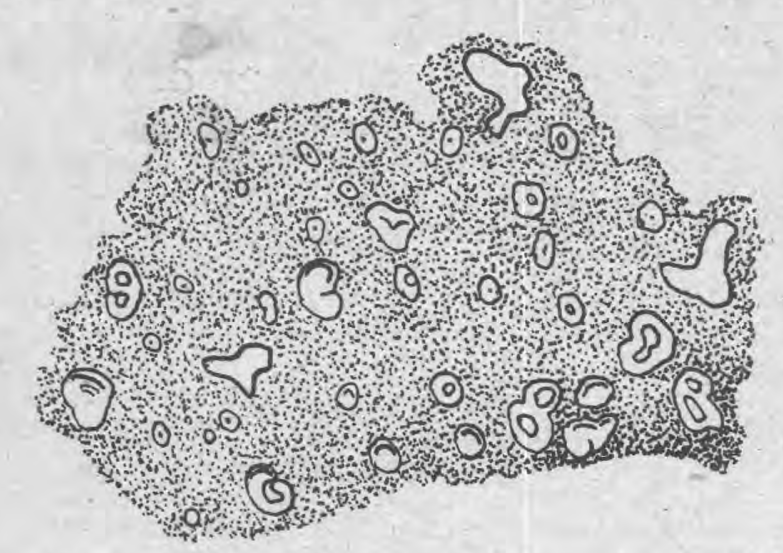

FIG. 32. - Fragment de fromage cheddar canadien pasteurisé de lait homogénéisé. Non coloré. Grossissement $t$ : 300 .

hétérogène. Ceei est d'une grande signification pour les fromages mous, qui subissent une telle falsification, spécialement pour le garmond, dans lequel on met très souvent de la caillebotte maigre de lait de vache. La garmond, ainsi que le fromage gras de brebis, légèrement mûri, a une pâte à gros grains, dans laquelle çà et là on trouve des grains de la leucine.

On ne peut jamais réduire si finement la caillebotte qu'on ne pourra pas reconnaître la falsification à l'observation microscopique. Dans un tel garmond ainsi falsifié, il se trouve distinctement des parties de la masse à grains fins (caillebotte maigre) (fig. 7).

Il est donc possible de compléter l'observation chimique des fromages dans plusieurs cas par un examen facile de la pâte au microscope, principalement dans les cas où les méthodes chimiques nous échappent, par exemple dans la falsification des fromages mous par la eaillebotte maigre, lorsqu'il s'agit d'une marehandise originale surchoix. L'examen microscopique de la pâte du fromage est aussi précieuse pour l'observation bactériologique dont le résultat doit concorder avec le résultat microcospique. 